\title{
Um Ambiente para Transmissão de Vídeos Instrucionais sob Demanda
}

\author{
Rafael Canan \\ André Luís Alice Raabe
}

\begin{abstract}
Resumo: Este artigo apresenta um ambiente para transmissão de vídeos instrucionais de apoio aos estudantes de disciplinas de graduação da UNIVALI, enfatizando o desenvolvimento do servidor de vídeo sob demanda que viabiliza a transmissão da mídia através de Streaming. O servidor foi desenvolvido utilizando a API Java Media Framework e é capaz de atender a vários clientes simultaneamente. Os arquivos são recuperados do servidor de mídia, são codificados e transmitidos através do protocolo RTP (Real Time Protocol). O desenvolvimento do servidor promoveu também uma integração do ambiente IMAGUS (Raabe \& Giraffa, 2001) com a ferramenta de autoria de páginas de ensino SAPENS (Kokubo \& Vavassori, 2003), criando um ambiente funcional de apoio ao uso de vídeos nas páginas de disciplinas de graduação na UNIVALI.
\end{abstract}

Palavras-chave: Streaming Vídeo; Vídeo sob Demanda; Sistemas Multimídia;

Title: An Environment for Transmition of Instructional Video on Demand

\begin{abstract}
This paper presents an environment for transmition of instructional videos to support students learning at UNIVALI undergraduate couses. It emphatises the vídeo on demand server development wich allows media streams to be delivered. The server was developed with Java Media Framework API and is prepared to respond to many cliente simultaneously. The media files are retrived from the media server, are coded an transmited using RTP (Real Time Protocol). The server development also promoted the integration between IMAGUS environment (Raabe \& Giraffa, 2001) and the authoring tool for website courses construction named SAPENS (Kokubo \& Vavassori, 2003), creating a functional environment to support videos at UNIVALI undergraduate couses websites.
\end{abstract}

Keywords: Vídeo Streaming; Vídeo on Demand; Multimedia Systems

\section{Introdução}

A utilização de vídeo como material didático ainda é realizada de forma muito tímida pelas instituições de ensino brasileiras. Acredita-se que dois fatores contribuem para que esta situação tenha se instalado: o despreparo dos educadores frente a utilização dos recursos audiovisuais como auxílio ao processo de ensino-aprendizagem, e os altos investimentos necessários para aquisição de infra-estrutura que permita uma utilização abrangente do recurso audiovisual na Educação.

Com o advento e expansão da Internet, surgiu uma alternativa para a disponibilização de vídeos com uma infra-estrutura reduzida. No entanto, devido a pouca largura de banda das redes atualmente em uso, o tráfego de informações multimídia ainda exige um esforço de investigação a fim de encontrar alternativas de baixo custo com qualidade suficiente para contemplar os objetivos pedagógicos.

Tendo em vista esta realidade, Raabe \& Giraffa (2001) propuseram um ambiente denominado IMAGUS, que buscou investigar a possibilidade de utilização de vídeo através da Internet como apoio a atividades para fins pedagógicos. O ambiente IMAGUS foi estruturado com o objetivo de possibilitar o desenvolvimento de aplicações educacionais que permitam a utilização de vídeos digitais em websites.

\footnotetext{
* Bacharel em Ciência da Computação - CTTMar - Universidade do Vale do Itajaí

** Doutorando em Informática na Educação, PGIE - UFRGS; Docente-Pesquisador e Coordenador do Grupo de Desenvolvimento de Software Educacional do Curso de Ciência da Computação - CTTMar - Universidade do Vale do Itajaí - SC - Brasil; E-mail: araabe@cttmar.univali.br
} 
Este artigo apresenta o mais recente avanço na arquitetura e implementação do ambiente IMAGUS, referindo-se a implementação de um servidor de vídeo que permite recuperar vídeos em um acervo e transmití-los aos clientes sob demanda, utilizando para isso o protocolo RTP (Real Time Protocol).

Apresenta-se também a integração de uma nova ferramenta de autoria, denominada SAPENS (Sistema Automático de Páginas de Ensino), ao ambiente IMAGUS. O SAPENS facilita a tarefa do docente de criar páginas Internet para disciplinas de graduação. A implantação do servidor de vídeo possibilitou que os docentes utilizem vídeos como material de apoio ao aluno nas páginas de suas disciplinas.

Não basta disponibilizar uma ferramenta aos docentes que permita o uso de vídeo, é preciso incentivá-los e orientá-los para tanto. Neste sentido, realizou-se um levantamento sobre os usos mais comuns de vídeo como recurso pedagógico que é apresentado na seção 2. Na seção 3 apresenta-se as tecnologias relacionadas com a transmissão de vídeo digital; na seção 4 apresenta-se o ambiente IMAGUS, a integração com o SAPENS o desenvolvimento do servidor de vídeo. A seção 5 apresenta as conclusões deste trabalho.

\section{O Vídeo como Material Didático}

- A imagem se tornou uma forma de comunicação extremamente relevante no contexto da sociedade atual. A mídia utiliza massivamente a imagem na publicidade, na televisão, em revistas e outros, a fim de comunicar fatos, idéias e conceitos.

As imagens em movimento, quando compostas de som (áudio) formam a informação denominada audiovisual. A utilização do recurso audiovisual na educação pode servir tanto no desenvolvimento de uma consciência crítica do educando frente a este tipo informação, quanto no suporte para alcançar objetivos pedagógicos específicos. Taddei (1981) denominou estas duas modalidades como: educar com a imagem e educar para a imagem. O ponto de encontro das duas modalidades é da imagem técnica, ou seja, a compreensão das técnicas de criação de audiovisuais.

Uma informação audiovisual é muito mais rica se comparada com uma informação textual, isso facilita a compreensão por parte dos alunos do material apresentado. Na visão dos alunos uma informação audiovisual está ligada a um contexto de lazer e entretenimento, que passa imperceptivelmente para a sala de aula. Para o aluno vídeo quer dizer descanso o que modifica a postura e as expectativas em relação ao seu uso. Educar com a imagem, representa tirar proveito do fato de que os alunos estão acostumados a linguagem visual permitindo reduzir o hiato de comunicação existente entre educando e educador.

Moran (1991) apresenta algumas formas de utilização do vídeo em sala de aula:

- Vídeo como sensibilização: Utilização do audiovisual para introduzir um novo assunto, despertar a curiosidade e a motivação para novos temas;

- Vídeo como ilustração: Por vezes a imagem ilustra melhor que as palavras. Esta modalidade permite trazer para sala de aula realidades distantes dos alunos como por exemplo da África ou Amazônia, permite a reconstituição de períodos históricos e outros;

- Vídeo como simulação: É uma forma mais sofisticada de ilustração. Permite simular experiência químicas perigosas, ou mostrar aceleradamente o desenvolvimento de uma árvore e assim por diante; 
- Vídeo como conteúdo de ensino: Quando o vídeo de direta ou indiretamente informa sobre um assunto específico orientando a sua interpretação e permitindo abordagens múltiplas;

- Vídeo como produção: Utilização das técnicas de produção de vídeos para documentação, intervenção e expressão de idéias de forma lúdica. Proporcionar aos alunos atividades de criação de audiovisuais;

- Vídeo espelho: visualização da auto-imagem buscando o autoconhecimento. Pode ser usado em grupo para análise dos papéis e comportamentos de seus integrantes;

- Vídeo como integração/suporte: Suporte de mídias como a televisão e o cinema através da exibição de filmes e programas gravados. Integração com outras mídias como o CD-ROM, vídeo-games e a Internet.

Segundo Roesler (2003), uma das áreas de Educação a Distância em foco atualmente é a de transmissão ao vivo de aulas pela Internet, considera-se esta mais uma modalidade interessante de utilização do audiovisual no suporte ao processo de ensinoaprendizagem.

Desta forma acredita-se que o uso do vídeo deve ser incentivado e explorado adequadamente como recurso potencializador da aprendizagem, e o desenvolvimento de ambientes computacionais auxiliem os docentes nesta tarefa estarão contribuindo para isso.

\section{Transmissão de Vídeo Digital via Internet}

A transmissão de vídeo através da Internet exige que se tenha uma largura de banda suficientemente grande para que a qualidade do vídeo a ser transmitido seja no mínimo razoável.

Devido às características das informações audiovisuais, uma aplicação multimídia na Internet, exige um alto desempenho da rede e das suas estações de trabalho. A videoconferência, por exemplo, só é válida quando lhe é oferecido o suporte capaz de garantir um grau de interatividade mínimo entre os participantes, sem prejudicar o andamento das outras aplicações da rede (Tanenbaum, 1997).

Para se ter uma noção do tamanho das informações audiovisuais, considere o vídeo como sendo uma seqüência de imagens paradas que apresentadas continuamente a um determinado intervalo de tempo, causam a impressão de movimento. Se cada imagem for de 300 x 200 pixels que contenha 60 kbytes de informações e levando em conta que a taxa de apresentação dos quadros de uma televisão é de 30 quadros por segundo, seriam necessários 8,9 minutos para transmitir os dados relativos a um segundo de vídeo utilizando um modem de 33,600 bps (Zanin, 1999).

Neste contexto a tecnologia streaming surgiu para possibilitar a transmissão de conteúdo multimídia sobre redes de pacotes de largura de banda reduzida e com reprodução em tempo real. O objetivo do streaming é estabelecer um método de entrega de um conteúdo para um determinado usuário em uma rede, e o aspecto básico da tecnologia é que a mídia é reproduzida a medida que os pacotes que à contém são recebidos, sem a necessidade de aguardar a transmissão de todo o arquivo.

Segundo Gomes (2002), no âmbito da tecnologias streaming, são normalmente utilizadas duas formas de distribuição de conteúdo pela rede:

- streaming sob demanda: os conteúdos permanecem armazenados em bibliotecas de servidores prontos para serem enviados sob requisição dos clientes (players). Estes arquivos são enviados via rede imediatamente 
após a sua requisição para serem reproduzidos. Este tipo de transmissão também é chamada de vídeo sob demanda (video on demand).

- streaming ao vivo: o conteúdo é veiculado de forma contínua, viabilizando a transmissão ao vivo e também a vídeo conferência.

Para a realização do streaming é necessário a utilização de protocolos de para o empacotamento e transporte da mídia através da rede. No caso de transmissões em tempo real a família de protocolos RTP, RTCP e RTSP estão entre os mais utilizados.

O Real Time Protocol (RTP) foi definido pela IETF RFC 1889 e é voltado para aplicações de tempo real, e pode ser utilizado para o envio de fluxos de áudio e vídeo ao vivo ou sob demanda. O RTP agrega além dos mecanismos para transporte da mídia um mecanismo suplementar referente ao controle dos pacotes enviados. Este controle é realizado pelo Real Time Control Protocol (RTCP). Tanto o RTP quanto o RTCP utilizam UDP para o transporte da mídia (Gomes, 2003).

Quanto ao tipo de comunicação entre o servidor da mídia e os clientes, tem-se as seguintes possibilidades: unicast (de um para um); multicast (de um para alguns); e broadcast (de um para muitos). No caso do vídeo sob demanda, a comunicação que ocorre normalmente é unicast, onde o servidor entrega a mídia para apenas um cliente.

Para o desenvolvimento de aplicações que realizem a transmissão de vídeo digital existem bibliotecas que facilitam a criação de sistemas para transmissão e reprodução da mídia. No ambiente IMAGUS adotou-se o JMF (Java Media Framework) para desenvolvimento dos componentes relacionados com a mídia, em especial o servidor de mídia apresentado neste artigo.

O JMF é uma interface para o desenvolvimento de aplicações voltadas a utilização de mídia temporal. Fornece suporte para as operações de aquisição, processamento e envio de mídia e suporta os principais formatos e codecs de áudio e vídeo existentes no mercado.

O JMF utiliza várias interfaces que definem o comportamento e a interação dos objetos utilizados para captura, processamento e apresentação da mídia. A implementação dessas interfaces opera com a estrutura do framework através de objetos intermediários conhecidos como gerenciadores.

O JMF utiliza quatro gerenciadores, sendo eles:

- Gerenciador (Manager): Controla a construção dos players e o processamento da mídias;

- Gerenciador de pacotes (PackageManager): Mantém um registro dos pacotes de mídia;

- Gerenciador de dispositivos de captura (CaptureDeviceManager) mantém um registro dos dispositivos de captura disponíveis;

- Gerenciador de plug-ins (PlugInManager): mantém um registro dos plug-in disponíveis, como os multiplexadores, demultiplexadores, CODECS entre outros.

Para desenvolver aplicações as quais utilizam a API JMF é necessário utilizar um destes gerenciadores, por exemplo, se a aplicação precisar capturar a informação audiovisual através de um dispositivo de entrada, será necessário utilizar o gerenciador de dispositivos de captura para encontrar qual dispositivo está disponível para acessar a informação.

\section{Ambiente IMAGUS}

O ambiente IMAGUS foi proposto por Raabe \& Giraffa (2001) para viabilizar o desenvolvimento de aplicações educacionais que utilizem vídeos através da Internet. A 
arquitetura do ambiente foi desenhada para auxiliar a tarefa dos docentes de disponibilizarem vídeos em páginas internet e viabilizar o acesso dos alunos a estes vídeos.

Foi desenvolvido um protótipo que demonstrou a viabilidade da arquitetura do ambiente, mas que não permitia a sua utilização em larga escala por docentes de uma instituição de ensino por apresentar problemas na ergonomia da tarefa a ser realizada.

Desta forma o ambiente foi redesenhado para contemplar a inclusão de uma ferramenta de autoria mais adequada e um servidor de vídeo funcional. A figura 1 apresenta a nova arquitetura do ambiente IMAGUS.

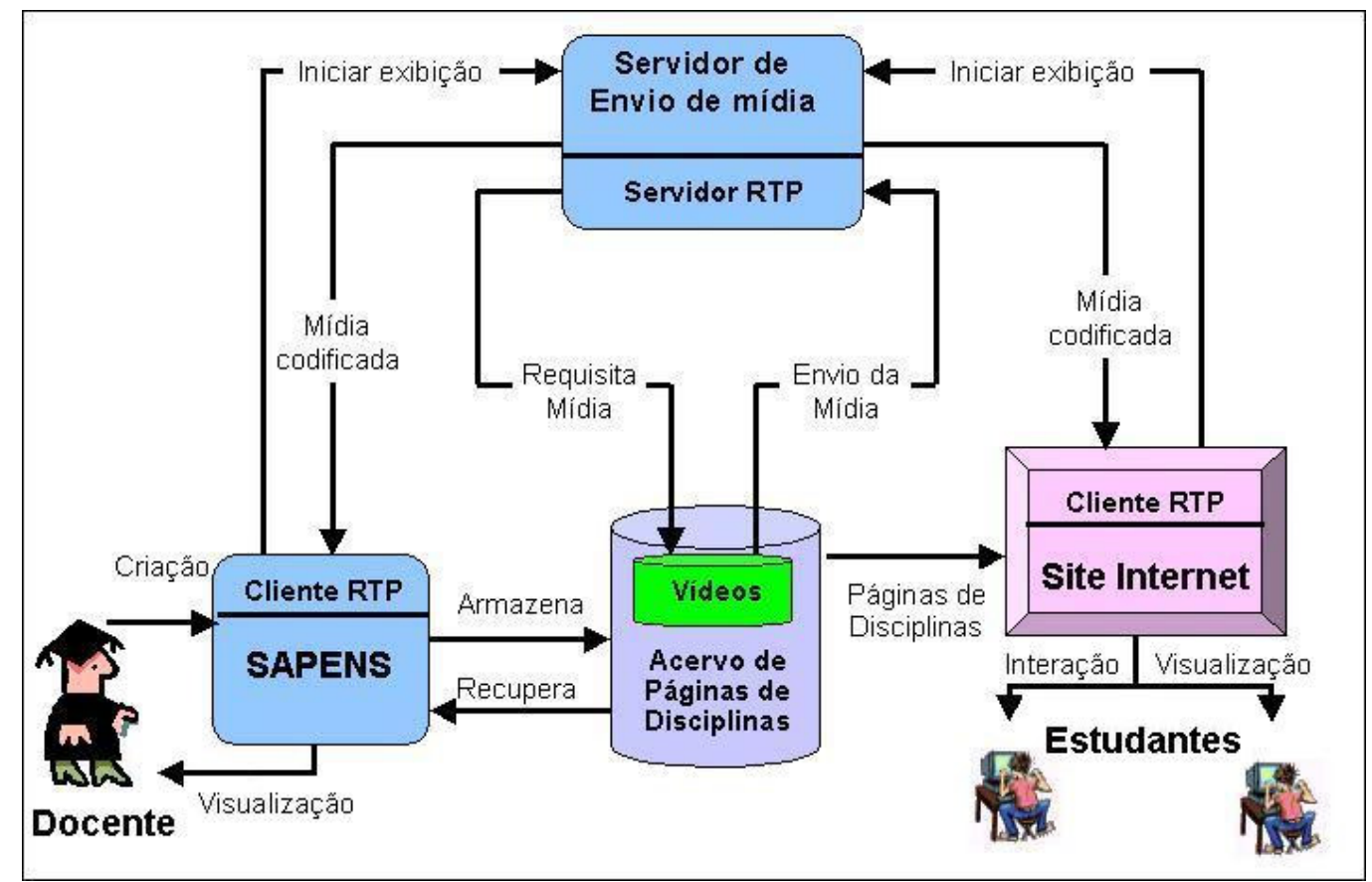

Figura 1 - Nova arquitetura do ambiente IMAGUS

De forma geral o ambiente possibilita que o docente elabore uma página para sua disciplina fornecendo informações básicas e os vídeos que deseja disponibilizar, o sistema SAPENS se encarrega então de gerar uma página padronizada e disponibiliza-a na Internet. $\mathrm{O}$ aluno ao navegar pelo website da disciplina visualiza a lista de vídeos que estão disponíveis e pode solicitar a reprodução. Neste momento é iniciado um cliente RTP que solicita ao servidor RTP que inicie o envio da mídia. Este por sua vez realiza a recuperação da mídia no servidor de mídia (banco de dados Oracle), codifica e transmite esta ao cliente que passa então a visualizar o vídeo.

A seguir são descritos os componentes do ambiente:

\section{SAPENS (Sistema Automático de Páginas de Ensino)}

De acordo com Kokubo \& Vavassori (2003), o SAPENS tem por objetivo facilitar a elaboração de páginas das disciplinas dos professores da UNIVALI na internet de forma rápida e simplificada sem que para isso os mesmos necessitem possuir um grande conhecimento no desenvolvimento de páginas para a web.

A escolha do sistema para compor a arquitetura do IMAGUS deve-se pelo fato de ser uma ferramenta já em uso e que demonstrou em suas primeiras versões a facilidade de uso e a eficácia na elaboração de páginas Internet para disciplinas de 
graduação. Além disso, o SAPENS possibilita a inclusão de materiais de apoio como arquivos para download, mas ainda não possuía suporte para a apresentação de vídeos.

A figura 2 ilustra a interface do SAPENS já com a funcionalidade de suporte a vídeos. A tela demonstra os vídeos inseridos pelo docente.

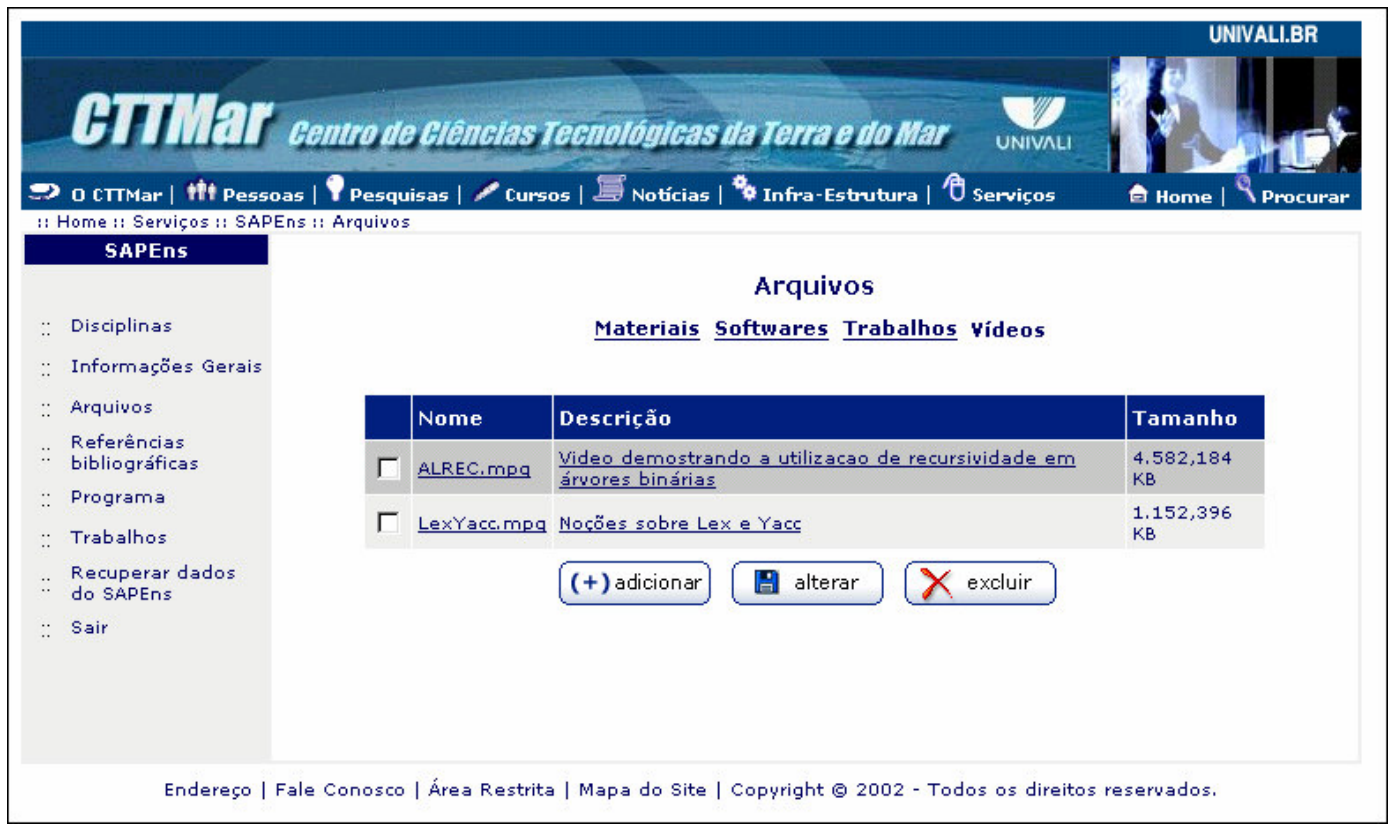

Figura 2 - tela de gerência de vídeos do docente

Acervo de Páginas de Disciplinas

Contém as informações inseridas pelos docentes através do SAPENS. São informações relativas ao conteúdo programático de suas disciplinas, materiais de apoio, apostilas para download, informações sobre trabalhos, avaliações etc.

$\underline{\text { Acervo de Vídeos }}$

Contém os vídeos inseridos pelos docentes para serem disponibilizados aos alunos. Os vídeos são inseridos também a partir do sistema SAPENS.

\section{Site Internet (cliente RTP)}

O site visualizado pelo aluno é gerado pelo sistema SAPENS de forma automática a partir das informações fornecidas pelo professor. Os vídeos disponíveis são apresentados ao aluno que pode seleciona-los para exibição. Neste momento, iniciase um applet Java contendo um player RTP que realiza a comunicação com o servidor para exibição do vídeo.

Servidor de Envio de Mídia (servidor RTP)

O servidor de envio de mídia realiza a recuperação da mídia junto ao servidor de mídia, instancia esta mídia em um arquivo temporário, codifica-a e transmite-a ao cliente utilizando o protocolo RTP.

Para desenvolvimento do servidor de vídeo sob demanda, um conjunto de aplicações e tecnologias foram integradas, dentre estas pode-se citar: PHP, Oracle, JDBC e JMF. A arquitetura interna do servidor de envio de mídia pode ser visualizada com mais detalhes na figura 3 . 


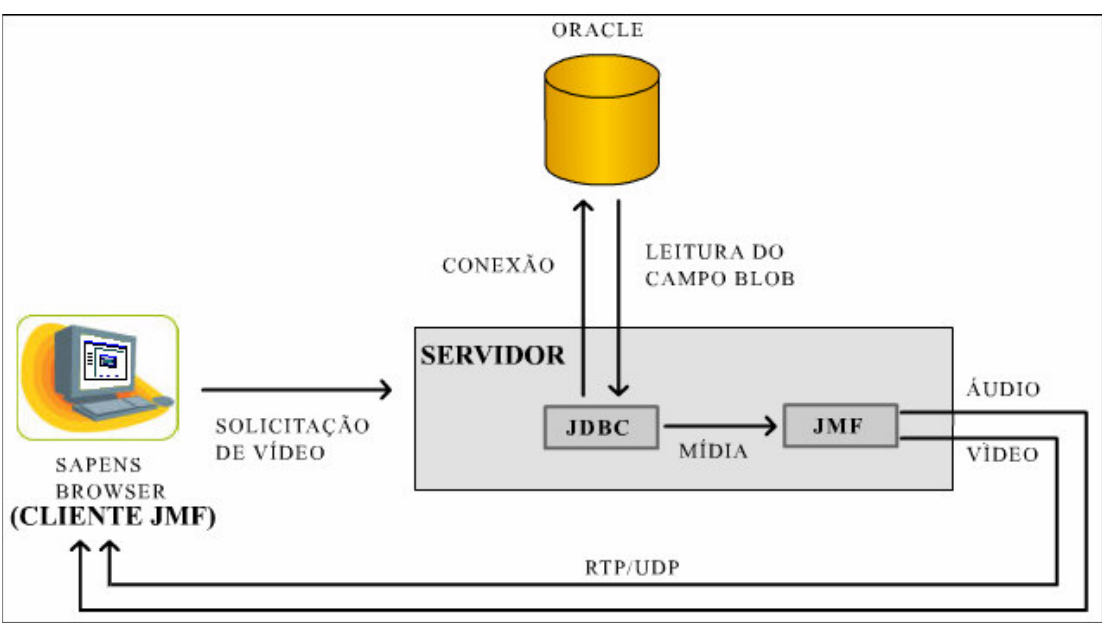

Figura 3 - Arquitetura interna do servidor envio de mídia.

Um usuário (aluno ou professor) acessa uma página web desenvolvida pelo docente através do SAPENS, e solicita a exibição do arquivo audiovisual o qual esta armazenado no acervo de vídeos. A solicitação é encaminhada para o servidor onde o mesmo utilizará a API JDBC para realizar as operações como conexão, pesquisa e recuperação dos dados armazenados no acervo de vídeos (Banco de Dados). Se o vídeo solicitado for encontrado o servidor começa a receber os dados do acervo, codificá-los e transmití-los por RTP através de outra API conhecida como JMF.

O servidor e o cliente negociam as portas para comunicação da seguinte forma. Inicialmente, o servidor possui um socket que fica aguardando as requisições dos clientes. Quando um cliente é executado, este estabelece uma comunicação com o servidor que valida a autenticidade do cliente e procura por duas portas disponíveis (uma para transmissão do áudio e outra para transmissão do vídeo) sendo que as mesmas devem possuir uma diferença de 2, como 6000 e 6002. Depois de encontradas no servidor, este pergunta ao cliente se essas mesmas portas estão disponíveis, caso contrário o servidor irá procurar por outras portas. Esse processo se repete até que o servidor e o cliente entrem em acordo ou até que termine o número de portas.

Foi construída uma interface para acompanhar o processamento realizado pelo servidor na comunicação com o cliente. A figura 4 ilustra o servidor processando a solicitação de um cliente.

Nenhum dos parâmetros internos do audiovisual como número de pixels, cores, o taxa de bits por segundo, ou frequiências sonoras são alterados ou comprimidos. O servidor apenas transmite o vídeo na forma como ele foi digitalizado.

Os componentes RTP (servidor e clientes) construídos através do JMF possibilitam automaticamente $\mathrm{O}$ monitoramento dos pacotes através do protocolo RTCP. Desta forma é possível identificar a qualidade da transmissão (atrasos, perdas, etc) a fim de ajustar os parâmetros de codificação do vídeo.

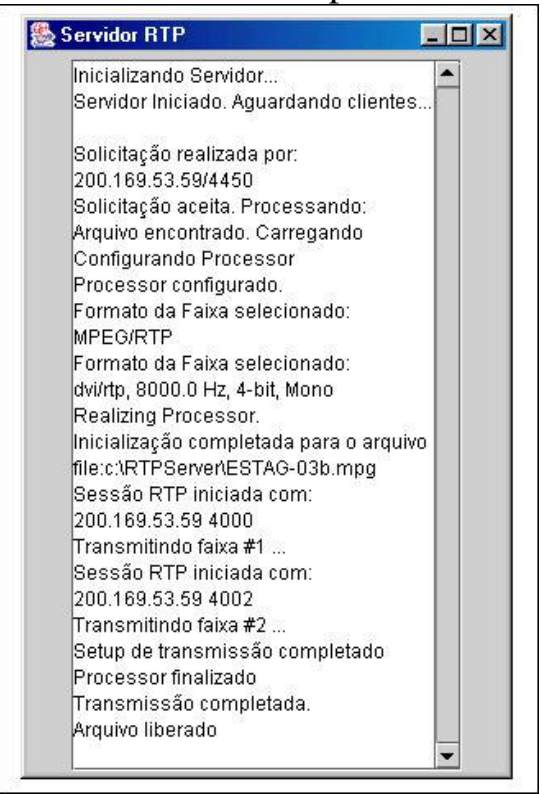

Figura 4 - Interface do servidor 
Infelizmente verificou-se impossibilidade da utilização do protocolo RTSP pelo fato do JMF não possuir suporte a esse protocolo na implementação de um servidor, isto acarretou na falta de controle do cliente sobre a reprodução do vídeo.

O servidor de vídeo é capaz de atender a varias solicitações simultâneas, limitando-se apenas pelo número de portas de comunicação disponibilizadas pelo administrador da rede.

\section{Conclusão}

Este artigo apresentou a nova arquitetura do ambiente IMAGUS enfatizando a implementação do servidor de vídeo sob demanda. A integração da ferramenta SAPENS e do servidor de Mídia proporcionaram mudanças na arquitetura original do ambiente proposta em Raabe \& Giraffa (2001). Certamente as mudanças aproximaram a arquitetura desenvolvida das tendências atuais, mas principalmente alteraram a concepção do acervo de vídeos que na idéia original era um acervo compartilhado entre os docentes, e na nova arquitetura é um acervo individual.

Os testes realizados com o ambiente ainda são preliminares, mas demonstraram a eficiência da inserção dos vídeos no sistema SAPENS e da exibição destes pelos clientes (professores e alunos). No entanto, todos os vídeos exibidos apresentaram um atraso médio de 2 segundos do áudio em relação ao vídeo. Até o momento não se descobriu o motivo de tal atraso. Verificou-se o funcionamento adequado do servidor com até 3 clientes simultaneamente, os testes com mais usuários não foram realizados por falta de um servidor com potência adequada.

Atualmente existem muitas alternativas proprietárias para o envio de mídia através da Internet, muitas delas com resultados excelentes. A intenção deste trabalho não é concorrer com as soluções de mercado, mas sim estabelecer uma plataforma que possibilite a experimentação de tecnologias a nível acadêmico, favorecendo assim a pesquisa e o desenvolvimento de aplicações integradas e adaptáveis. 


\section{Referências Bibliográficas}

GOMES, FÁBIO L. S. Videoconferência: Sistemas e Aplicações. Visual Books, Florianópolis, 2003.

IETF RFC 1889. Internet Enginnering Task Force - RTP A Transport Protocol for Real-Time Application. $1996 . \quad$ Disponível em: http://www.ietf.org/rfc/rfc1889.txt?number=1889. (Acessado em 21 abr 2003).

KOKUBO, Eduardo; VAVASSORI, Fabiane Barreto. Sistema Automático de Páginas de Ensino. Congresso Brasileiro de Computação 2003 CBCOMP-2003.

MORAN, José Manuel. Como ver Televisão - leitura crítica dos meios de comunicação. São Paulo: Ed. Paulinas 1991.

RAABE, ANDRÉ L. A.; GIRAFFA, LÚCIA M. M. Ambiente para Construção de Materiais Instrucionais Apoiados por Vídeo. Revista Brasileira de Informática na Educação, Florianópolis - SC, v. 8, p. 1-13, 2001.

ROESLER, VALTER; ANDRADE, MAIKO DE; TAROUCO, LIANE; On-line remote class with live video transmission: a study case. Computers and Advanced Technology in Education Conference / Web Based Education - CATE/WBE 2003. Rhodes. Grécia.

TADDEI, Nazareno. Educar com a Imagem. Voume II. Edições Loyola. São Paulo, 1981.

TANENBAUM, A. Redes de computadores. Rio de Jan.: Campus, 1997. 923 p.

ZANIN, Fabio A.; ALMEIDA, Maria Janilce B. O ambiente de videoconferência sobre redes ATM. Trabalho Individual - CPGCC-UFRGS. Porto Alegre, Jan. de 1999. 